\title{
Research on high-temperature heat receiver in concentrated solar radiation system
}

\author{
Przenzak Estera ${ }^{1, *}$, Basista Grzegorz ${ }^{1}$, Bargiel Paulina ${ }^{1}$, Filipowicz Mariusz ${ }^{1}$ \\ ${ }^{1}$ AGH University of Science and Technology, Faculty of Energy and Fuels, Department of Sustainable Energy Development, A. \\ Mickiewicza Av. 30, 30-059 Kraków, Poland
}

\begin{abstract}
The article presents the results of experimental and computer simulations studies of the high temperature heat receiver working in the concentrated solar radiation system. In order to study the radiation absorption process and heat exchange, the two types of computer simulations were carried out. The first one was used to find the best location for absorber in the concentrating installation. Ray Tracing Monte Carlo (RTMC) method in Trace Pro software was used to perform the optical simulations. The results of these simulations were presented in the form of the solar radiation distribution map and chart. The data obtained in RTMC simulations were used as a second type boundary conditions for Computational Fluid Dynamics (CFD) simulations. These studies were used to optimize the internal geometry of the receiver and also to select the most effective flow parameters of the working medium. In order to validate the computer simulations, high temperature heat receiver was tested in experimental conditions. The article presents the results of experimental measurements in the form of temperature, radiation intensity and power graphs. The tests were performed for varied flow rate and receiver location. The experimental and computer simulation studies presented in this article allowed to optimize the configuration of concentrating and heat receiving system.
\end{abstract}

\section{Introduction}

Nowadays, great emphasis in each of the activities is put on sustainable development. One of its most important aims is to ensure affordable and clean energy. There are many different ways to gain energy from resources that meet high demands from both ecological and economical sides.

Among all renewable resources more and more attention is paid to solar energy, which huge potential still remains untapped. Energy gained from the Sun is widely accessible and may be easily converted into for e.g. heat or electricity. Moreover, it is commonly use in small and medium-small buildings as a source of cheap and safe energy. There are two main application used to obtain energy from solar radiation. Solar thermal collectors receive heat for domestic applications and photovoltaic (PV) technologies produce electricity. It is also possible to connect this both systems into one called hybrid photovoltaic-thermal system, what improve their performance. However, the power density of energy obtained from the solar radiation is relatively low compared to other energy sources. Due to this disadvantage it is important to develop various kinds of concentrating systems, which provide amplification of the intensity of solar radiation incident on the surface of the receiver.

Preparing a properly operating installation requires performing a series of simulations. For solar systems, it is necessary to analyse the calculation of energy flux distribution on the receiver. One of the widely used technique is a ray tracing simulation. This universal tool can be exploit to survey reflection, refraction, diffraction and scatter of the light beams. In [1] ray tracing method allows to perform the simulation of the optical behaviour of three cavity receivers of various geometry complexity (spherical, cylindrical and conical). Ray tracing was also used to simulate a multi-faceted point focus concentrator composed of 18 spherical mirrors [2]. Due to this model it was available to achieve a homogenous flux distribution on the surface of the receiver. Moreover, in [3] has been presented a project of a solar concentrator, wherein the heat is transferred by bundled optical fibre instead of a heat receiver [3]. The simulation was performed using modified ray tracing method so-called Monte Carlo, in which the rays are randomly defined according to a probability density.

Computational Fluid Mechanics (CFD) plays a significant role in analysis of complex energetic processes. In case of solar heat receivers, due to CFS simulations some of the crucial operational parameters can be obtained, for e.g. heat transfer or distribution of the temperature on the absorbing surface. This method is widely used and very popular because of achieving good simulation results in compare to taken measurements. In [4] solar volumetric - receiver for high temperatures was designed using CFD calculations. The results obtained on the basis of tests carried out on the experimental

*orresponding author: esterap@agh.edu.pl 
prototype and CFD model proved to be very similar, the temperature differences between the measurements and CFD simulations was from 3.2 to $8.4 \%$, which is an acceptable result. Solar cavity receiver used to transfer the energy absorbed from solar radiation to the working medium in solar tower system [5] was simulated using CFD method. Simulation results allowed to perform optimisation of surface heat flux distribution inside the receiver, especially its uniformity. Influence of porosity on the thermal analysis and design of the solar received was studied in [6]. Temperature and velocity distribution were simulated using CFD software in terms of various configurations of the absorber. Due to porous structure (constant and gradual porosity) used material feature highly resistant to thermal stresses, but the thermal efficiency depends strongly on the distribution and depth of pores.

\section{Description of the solar system}

At the Department of Sustainable Energy of the AGH University of Science and Technology, research on the hybrid solar energy cooling system is conducted. The system consists of solar radiation concentrator combined with a classic solar collector system. The aim of the research is verification of the possibility of cold generation during high demand periods. Main components of the system installed on the roof of the D9 building (the Department of Sustainable Energy of the AGH University in Kraków) are: a parabolic solar radiation concentrator, which reheats the working medium (thermal oil) flowing between the solar collectors and the tank [7], two flat solar collectors connected to a 1501 buffer tank.

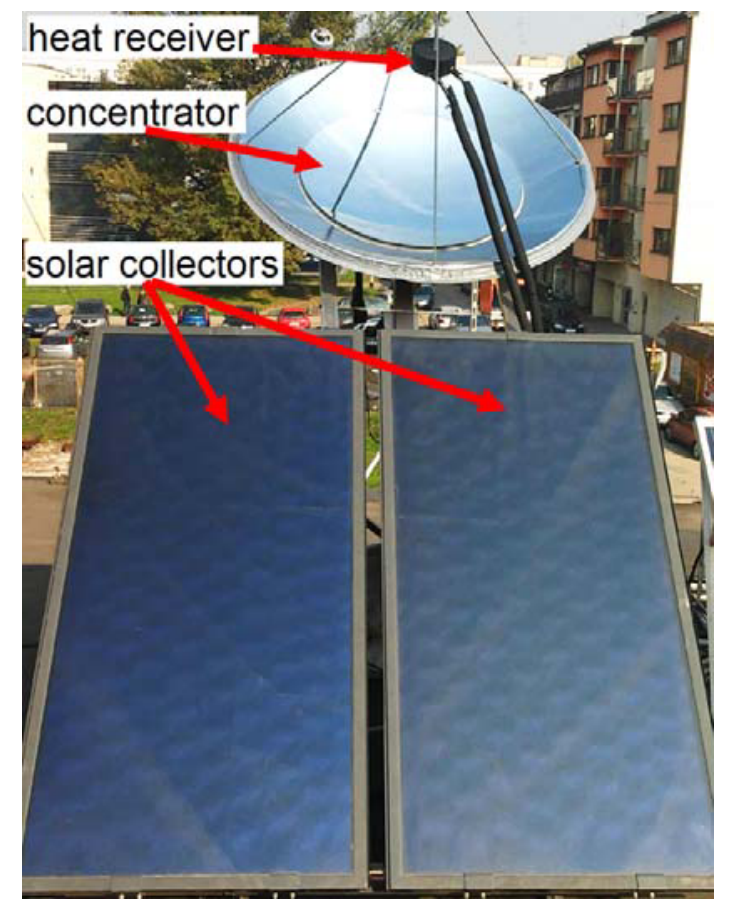

Fig. 1 View of the hybrid system of solar panels and solar radiation concentrator mounted on the roof of Department of Sustainable Energy Development, AGH University in Krakow.
Due to safety reasons, the system has been equipped with a dry-cooler protecting again overheating of the collectors and the utility water tank. It allows for conducting long-term research. Ultimately, the entire system shall be equipped with an absorption chiller. Curretly, efficiency of high temperature heat generation in term of absorption chiller driven is in progress.

\subsection{Solar radiation concentrator}

The solar radiation concentrator used in the research has been composed of two concentrating elements: A dish covered with a two-layer reflective foil $(1.8 \mathrm{~m}$ in diameter) and a mirror mounted on the dish with a diameter of $1.2 \mathrm{~m}$. The system in concern is characterized by two focal points of rays (in the distance of $70 \mathrm{~cm}$ and $85 \mathrm{~cm}$ from the mirror's surface), which results from the different geometries of the focusing elements and the fact that the mirror has been installed at a distance of $2 \mathrm{~cm}$ from the dish surface. The concentrator's system follows the location of the sun using a differential sensor and two linear actuators. This solution enables the changing of the azimuth and elevation angle in automatic mode.

\subsection{High-temperature heat receiver}

An important element of the installation in terms of generation of high-temperature heat is a receiver, which is placed in a focal point of concentrated solar radiation. The main impact on radiation absorption efficiency has the location and shape of the receiver. In turn, the efficiency of high-temperature heat removal by working medium depends on the inner geometry of the receiver. The study has been conducted on a flat heat receiver with a diameter of $18 \mathrm{~cm}$, which absorbing surface was covered by high-temperature black paint. Back and side walls of the receiver were insulated using mineral wool with a thickness of $2 \mathrm{~cm}$. The receiver comprised of a aluminium block with milled internal canal, a cover, a seal and stub-pipes.

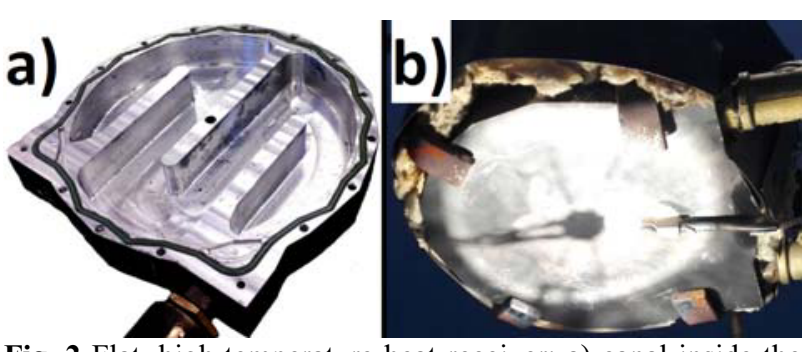

Fig. 2 Flat, high-temperature heat receiver: a) canal inside the receiver, b) receiver lighted by concentrated radiation.

In figure $2 \mathrm{a}$ the interior of the receiver is shown. The working medium canal, a seal, screw used to fasten the cover and one of the stub-pipe are visible. Figure $2 b$ presents the receiver operating in concentrated solar radiation. A focused rays falling on the absorbing surface and also a mineral wool insulating against heat loss are visible. 
Currently, receivers with concave and convex surfaces are tested. However, this article presents the results related only to the flat receiver, which features the simplicity of the construction and flawlessly exploitation.

\subsection{Measurement and control system}

The installation was equipped with the automatic control and measurements system based on the programmable logic controller (PLC). A number of measurement elements, like sensors of temperature, pressure, solar radiation intensity, wind and a flow meter are connected to the controller. The $370 \mathrm{~W}$ oil pump, controlled by the inverter, is used as a circulation pump. Moreover, the automatic system allows to control the performance of the cooler fan used as a protection against overheating of the installation.

The installation is equipped with 10 temperature sensors installed in important points of the system together with 8 solar radiation intensity sensors. Detailed description of the system is presented in paper [8].

Experimental studies of the hybrid installation were conducted during the summer period. The cloudless, characterized by low intensity of the scattered radiation were selected. A series of measurements of the energy parameters was conducted for 10 different locations of the receiver in the geometrical system of the concentrator. The distance of the receiver from the surface of the mirror was changed every $2 \mathrm{~cm}$ from 72 to $90 \mathrm{~cm}$. For each selected distance, the power of circulation pump was varied. The relative pump capacity was changed every $5 \%$ in the range of $80 \%-60 \%(55 \%$ if the flow was still too high). In this way the temperature variation was examined for medium flow from approx. 1,4 to 4,7 1/min. Each single measurement (the given distance, pump capacity) took approx. 20min necessary for the system stabilisation.

\section{Experimental research}

All raw measured points are collected in general figure (fig.3) in temperature difference - flow coordinates. In further part of the paper detailed analyses including also data form solar radiation sensors is presented.

As shown in figure 3 , the highest temperature difference was achieved for two distances of the receiver, i.e. for $76 \mathrm{~cm}$ and $78 \mathrm{~cm}$. For low medium flows of approx. 1.4-3 1/min the temperature difference was achieved at the level of 12-20 . This value is sufficient to increase the temperature of the working medium behind solar collectors to the values necessary for the effective operation of an absorption cooler.

The change of the distances to 72, 74, 80 and $82 \mathrm{~cm}$ causes a decrease the temperature differences to the level of approx. 8-15. However, these values can also be sufficient for powering the absorption chiller, but with lower efficiency.

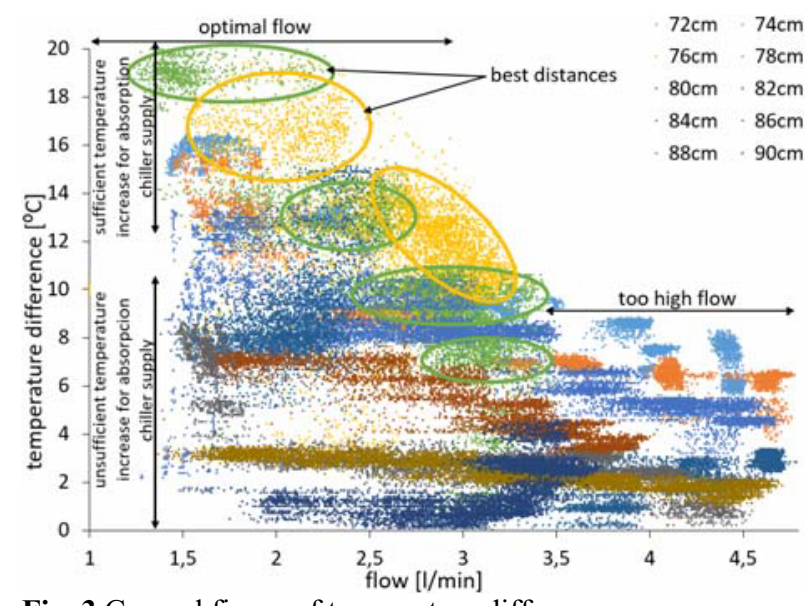

Fig. 3 General figure of temperature difference

The worst results (less than 8 ) were achieved for distances $84-90 \mathrm{~cm}$. It can be seen that the change of the flow has a very slight effect on the temperature increase. At the same time, we can see that the flows over $3.51 / \mathrm{min}$ for each location of the receiver are too high for the effective heat generation.

\subsection{Temperature variation}

The temperatures measured at selected parts of the installation depend on the circulation pump capacity, as is shown in fig 4.

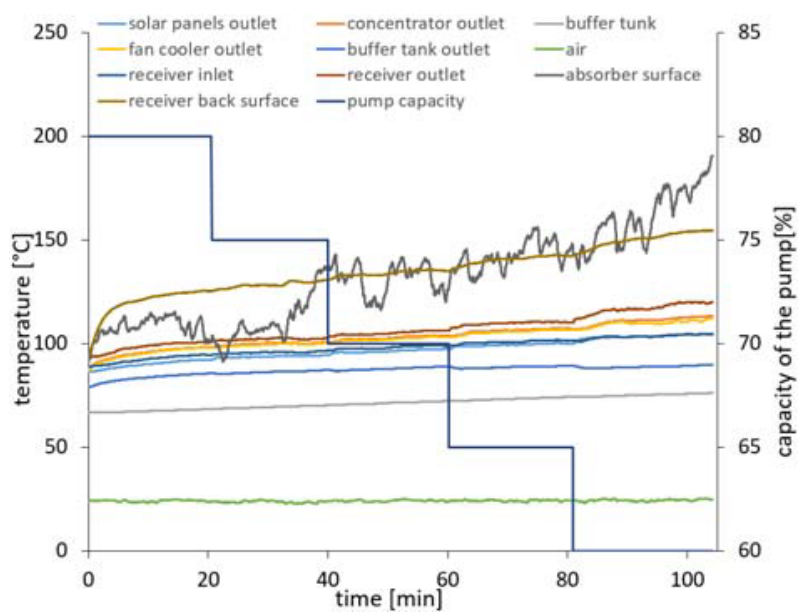

Fig. 4 Graph of the temperatures variation in selected points of the installation.

The increase of the working medium temperature is observed with the decrease of the flow. It is also seen, that the temperature on the absorber surface changes quite rapidly. It is caused by the inaccuracy of the solar tracking system. The focal point of concentrated radiation escapes from the central part of the receiver. This is one of the problem associated with the use of concentrated solar systems. It should be included that a sensor positioned on the absorbing surface (seen on fig.2b) is additionally heated by the concentrated solar radiation. 


\subsection{Solar radiation intensity}

In fig.5 the solar radiation variation registered by five sensors corresponding to the temperature measurements presented in fig. 4 is shown. In approximately 35 minute a rapid change of the direct radiation intensity, caused by the inaccuracy of the solar tracking system is visible. It can be seen that the measurement was carried out in a sunny, cloudless day - that is a cause of low values of scatter radiation and the lack of dynamic changes of the radiation intensity.

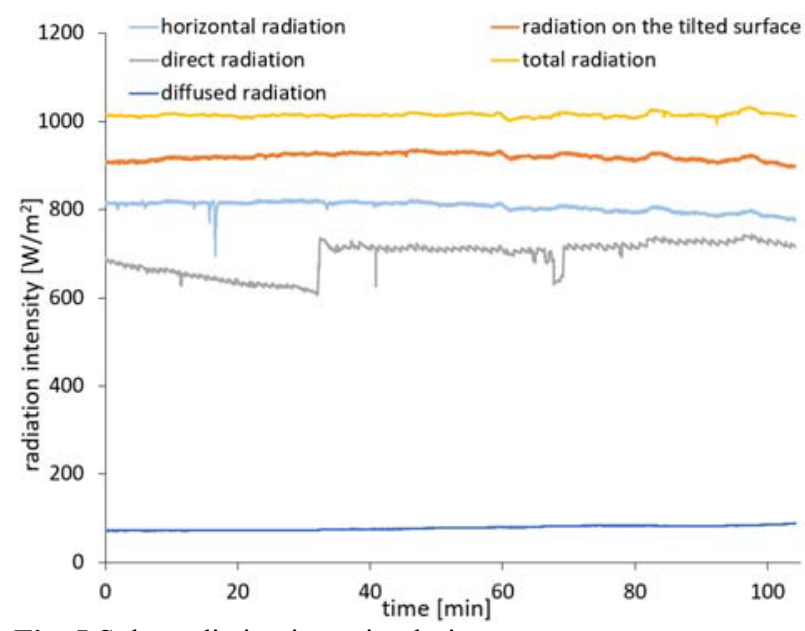

Fig. 5 Solar radiation intensity during measurements.

\subsection{Power of the receiver}

Based of the registered data, the power of the receiver (considered as a heat source) was calculated. The results are shown in fig. 6.

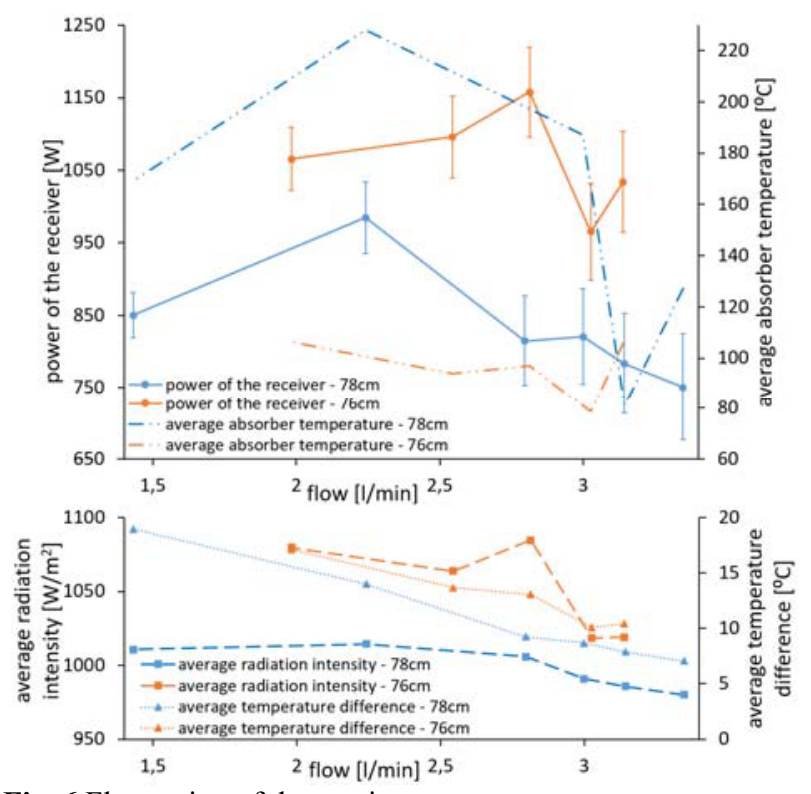

Fig. 6 Fluctuation of the receiver power

It can be seen that in the case of the receiver located at a distance of $78 \mathrm{~cm}$ from the mirror surface, the maximum power was achieved for the flow with a value of approx. $2.31 / \mathrm{min}$. Lower flow (1.51/min) causes the loos of the power even despite achieving a large temperature difference (approx. 20 ).
The maximum power for the distance of $76 \mathrm{~cm}$ was achieved for the flow approx. 2.81/min. However, it should be noted, that during the flows higher than $2.51 / \mathrm{min}$, and therefore for the pump efficiency in the range of $65-60 \%$, a significant dynamic changes of the solar radiation intensity happened (fig.7).

In the graph fig. 7 one of the basic disadvantages of the systems using the concentrated solar radiation can be observe. Namely, it can work effectively only on sunny cloudless days.

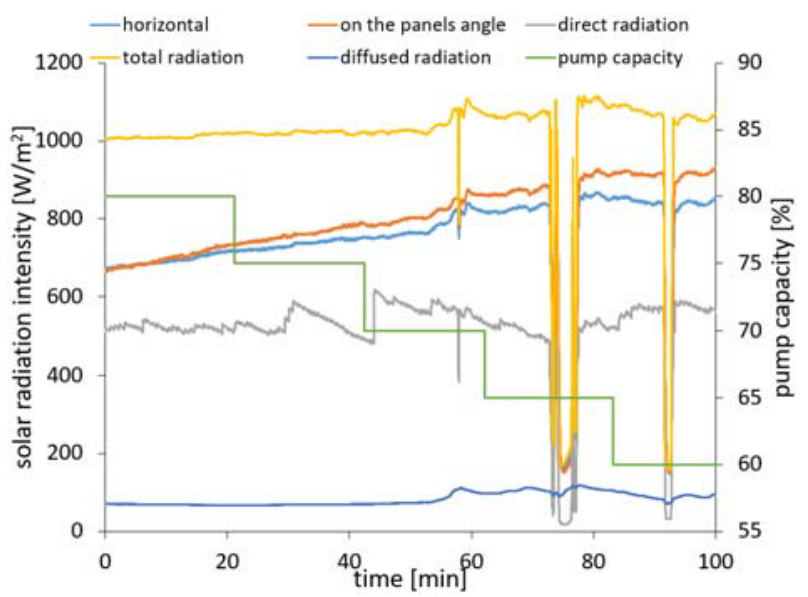

Fig. 7 Solar radiation intensity for measurements at distance of $76 \mathrm{~cm}$

A visible decrease in solar radiation intensity has a significant impact on the achieved value of the receiver's power. Comparing the two distances, it can be noted that the receiver located closer to the mirror $(76 \mathrm{~cm})$ achieves an output almost $200 \mathrm{~W}$ higher compared to the receiver moved $2 \mathrm{~cm}$ back. In some part it is effect of the higher average radiation intensity comparing intensity for the distance $78 \mathrm{~cm}$. This effect will be included in computer simulations presented in the further part of the paper.

\section{Computer simulations}

To explain observed data, the computer simulations were carried out. For this purpose, two simulation methods were used. In the first one, the Ray Tracing Monte Carlo (RTMC) method was applied to trace solar rays in the optical system. The second method - Computational Fluid Dynamics - was used for creating the model of the working medium flow inside the high-temperature receiver.

\subsection{Ray Tracing}

The Ray Tracing method was used to determine energy of solar radiation delivered to the surface of the receiver in function of the distance from the parabolic mirror. [9]. On this basis the optimal distance was selected $-76 \mathrm{~cm}$. Computer simulations were made using the software of the Lambda Research company [10].

For the purposes of conducting the simulation, 4 geometric blocks were designed: a canopy and a concentrator's mirror, flat receiver of the hightemperature heat and the Sun. For the bowl covered with 
reflective foil, the reflectance ratio was set at the level of $70 \%$, while for the mirror surface this rate was set to $95 \%$. The absorbency rate was adopted for the absorption surface of the receiver at the level of $90 \%$. An important parameter is also the spectrum of solar radiation emitted by the block of "the Sun". The spectrum in conformity with the standard ASTM E-49000 was used [11].

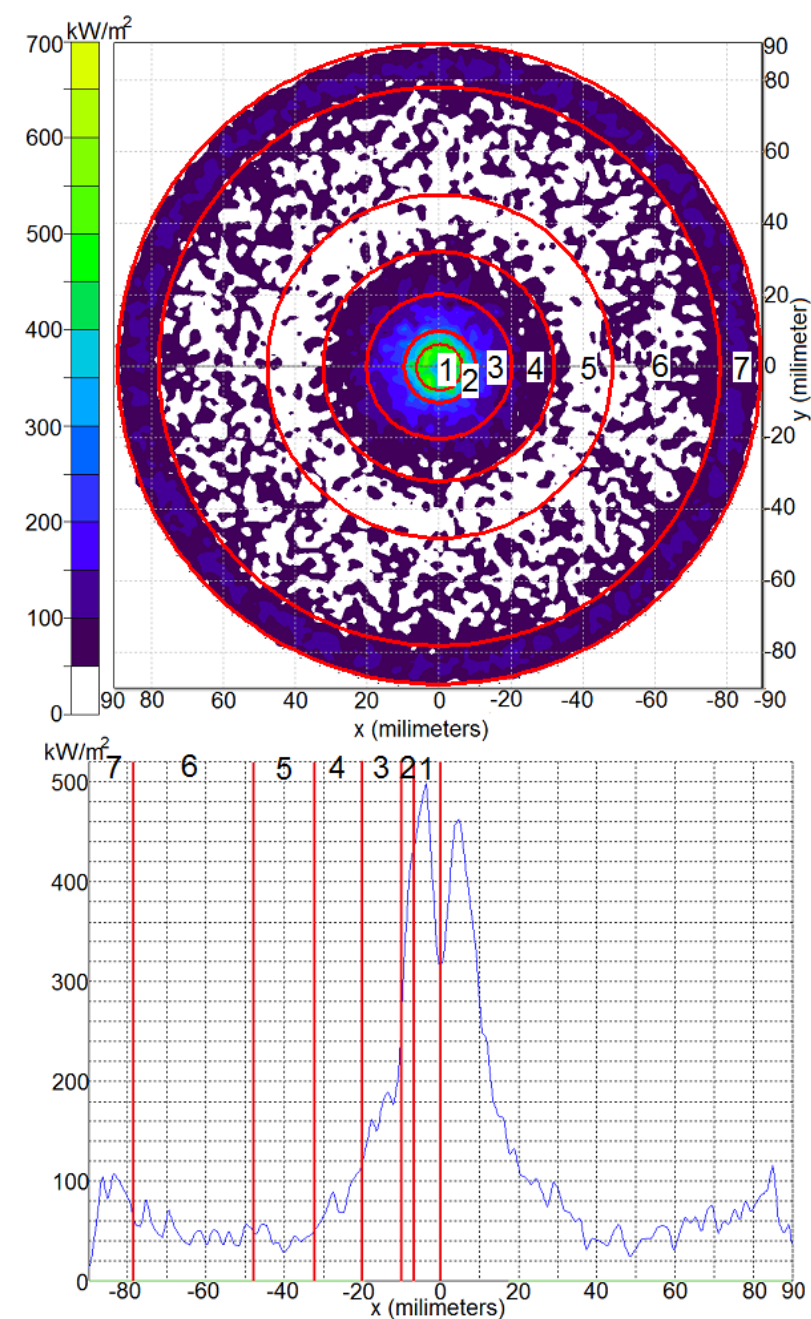

Fig. 8 Map and graph of radiation intensity on the absorbing Surface of high temperature heat receiver.

The values of the solar radiation intensity on the absorption surface were generated in the form of maps [9]. Fig.8 presented a sample map for a distance of $76 \mathrm{~cm}$, where the intensity of radiation emitted from the surface of the Sun was $1025 \mathrm{~W} / \mathrm{m}^{2}$.

In fig. 8 it can be seen that the intensity of solar radiation on the entire absorption surface of the receiver is not uniform. The highest intensity was noted on a small area (less than $1 \mathrm{~cm}^{2}$ ) in the central part of the receiver - approx. $600-700 \mathrm{~kW} / \mathrm{m}^{2}$. The largest part of the receiver absorbs the radiation with the intensity of approx. $50-100 \mathrm{~kW} / \mathrm{m}^{2}$.

The data obtained through the simulation using the Ray Tracing Monte Carlo method was used for creating a numerical model of the working medium flow inside the receiver using the Computational Fluid Dynamics method. For this purpose, the surface of the receiver was divided with circles presented in fig. $\mathrm{H}$. The average values of the radiation intensity in each marked ring were used as the value constituting the boundary condition of the second type in the CFD modelling.

Tab. 1. Boundary conditions for RTMC model

\begin{tabular}{|c|c|c|c|c|}
\hline \multicolumn{4}{|c|}{ Experiment } & $\begin{array}{c}\text { RTMC } \\
\text { model }\end{array}$ \\
\hline $\begin{array}{c}\text { flow } \\
{[1 / \mathrm{min}]}\end{array}$ & $\begin{array}{c}\text { receiver } \\
\text { inlet } \\
\text { temp. }\end{array}$ & $\begin{array}{c}\text { receiver } \\
\text { outlet } \\
\text { temp. }\end{array}$ & $\begin{array}{c}\text { average } \\
\text { radiation } \\
\text { intensity } \\
{\left[\mathrm{W} / \mathrm{m}^{2}\right]}\end{array}$ & $\begin{array}{c}\text { total flux } \\
\text { on the } \\
\text { absorber } \\
\text { [W] }\end{array}$ \\
\hline 3.1 & 55.6 & 66.2 & 1020 & 1733 \\
\hline 2.9 & 60.2 & 70.3 & 1025 & 1743 \\
\hline 2.7 & 64.9 & 77.5 & 1081 & 1838 \\
\hline 2.5 & 56.9 & 67.4 & 1020 & 1733 \\
\hline 2.2 & 74.2 & 92.6 & 1077 & 1831 \\
\hline
\end{tabular}

For the distance indicated on the path of the computer simulations of the distances of $76 \mathrm{~cm}$ five experimental measurements were selected with a different value of the working medium flow. The intensity of the solar radiation in the simulation was set as a value read from the measurement transducers during the experimental research. As a result of the conducted analyses, based on the results of the simulations, the total flux absorbed by the receiver's surface was calculated. The results are presented in table 1.

\subsection{Thermal analysis}

The experimental studies have allowed the measurement of the temperature difference of the working medium between the outlet and inlet to the device. The spatial numerical model using the computational fluid dynamics model (CFD) was developed for a more detailed way of analysing the operation of the device and location of the potential imperfections. The model describes the phenomena related with the flow of the working medium, and with the heat exchange between the receiver and the environment. The model in the steady state was made using the commercial software ANSYS Fluent [12].

In the first stage of works, the spatial geometry was prepared, representing the real receiver and the domain of liquid filling it. Then, the computing grid was prepared, for the purposes of the numerical model. After the final adjustment, it consisted of about 3,1 million elements, mostly hexagonal in nature. The boundary layer consisted of 8 layers with the growth factor equal 1.2 , and height of the first element equal $0.25 \mathrm{~mm}$. The cross-section of the computational grid of the liquid domain with the visible boundary layer was presented in fig.9.

The material of the fluid domain was defined using data of the thermal oil manufacturer. The properties of the oil, such as specific heat, density and viscosity were defined as the temperature functions, using higher order polynomials. The domain material of the receiver was aluminium, which properties were defined based on the database of the program. 


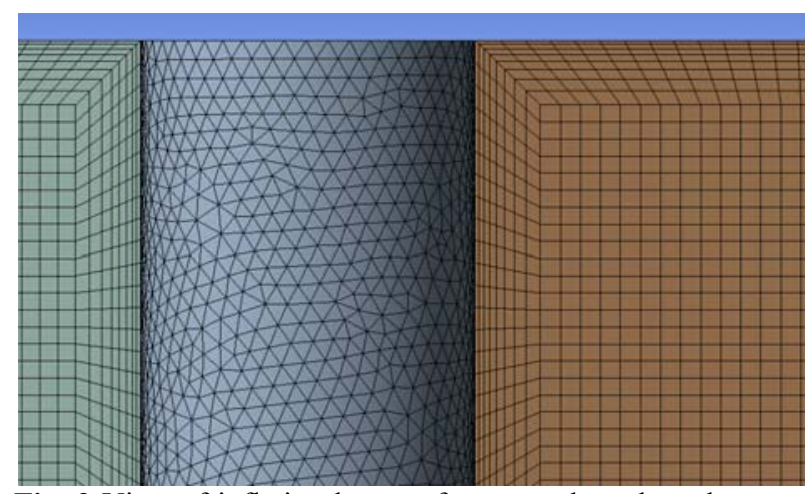

Fig. 9 View of inflation layers of generated mesh at the cross section of fluid domain

2-equation turbulence model SST k-omega was used for the calculations. The contact surfaces between the domains were described using the two-directional interfaces.

The constant boundary conditions of the model were the relative pressure at the outlet of the domain, equal 0 $\mathrm{Pa}$, and parameters of the heat exchange on the rear and side walls of the receiver. The heat transfer coefficient was defined as $10 \mathrm{~W} /\left(\mathrm{m}^{2} \mathrm{~K}\right)$, while the coefficient of emissivity of the surface was equal 0.85 . These conditions were fixed for each of the calculation cases. However, the real conditions of the environment during individual measurement series of the experimental studies were variable. This may have a slight impact on differences between the results of the model and the experiment.

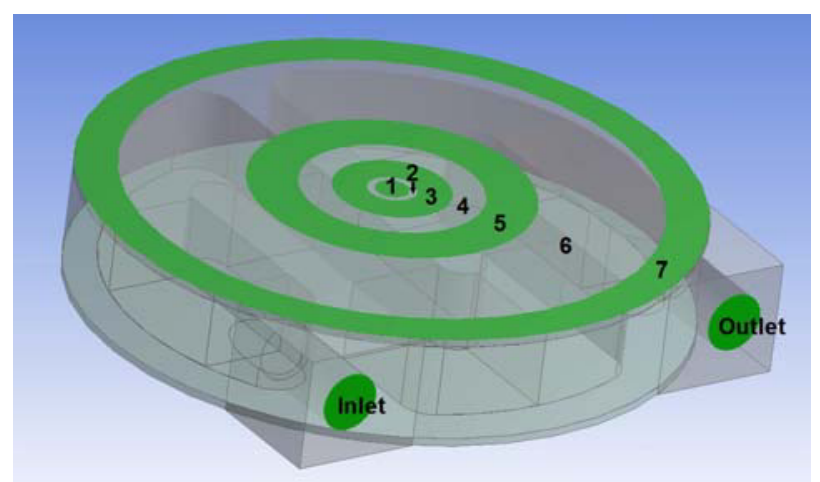

Fig. 10. Surfaces for boundary conditions

The remaining boundary conditions were defined using the parameters in order to perform the variant analysis. Six cases (DP0 - DP5) with different boundary conditions defined based on the experimental and RTMC data, have been developed, to prepare devices characteristic. The full set of boundary conditions for all design points was presented in the table 2 . The areas, on which the boundary conditions were defined for each case, on the other hand were presented in fig. 10. The areas 1-7 on the absorber's surface correspond the boundary conditions "In1-In7", while "Inlet" and "Outlet" are the inlet and outlet boundary conditions for the fluid domain. For each case also the pressure in the system was defined, based on the results of the experimental research.
Tab. 2. Boundary conditions for design points DP0-DP5

\begin{tabular}{|c|c|c|c|c|c|}
\hline DP0 & DP1 & DP2 & DP3 & DP4 & DP5 \\
\hline \multicolumn{6}{|c|}{ Inlet Velocity $[\mathrm{m} / \mathrm{s}]$} \\
\hline 0.130 & 0.144 & 0.164 & 0.177 & 0.190 & 0.200 \\
\hline \multicolumn{6}{|c|}{ Inlet Temperature [K] } \\
\hline 371.0 & 347.3 & 331.6 & 338.0 & 330.0 & 328.7 \\
\hline \multicolumn{6}{|c|}{ Operating Pressure $\left(\cdot 10^{-5}\right)[\mathrm{Pa}]$} \\
\hline 1.76 & 1.76 & 1.75 & 1.76 & 1.80 & 1.78 \\
\hline \multicolumn{6}{|c|}{$\operatorname{In} 1\left[\mathrm{~kW} / \mathrm{m}^{2}\right]$} \\
\hline 294 & 308 & 301 & 301 & 294 & 287 \\
\hline \multicolumn{6}{|c|}{ In $2\left[\mathrm{~kW} / \mathrm{m}^{2}\right]$} \\
\hline 224 & 259 & 266 & 273 & 259 & 252 \\
\hline \multicolumn{6}{|c|}{$\operatorname{In} 3\left[\mathrm{~kW} / \mathrm{m}^{2}\right]$} \\
\hline 112 & 126 & 126 & 126 & 126 & 119 \\
\hline \multicolumn{6}{|c|}{ In4 $\left[\mathrm{kW} / \mathrm{m}^{2}\right]$} \\
\hline 49 & 56 & 56 & 56 & 56 & 56 \\
\hline \multicolumn{6}{|c|}{$\operatorname{In} 5\left[\mathrm{~kW} / \mathrm{m}^{2}\right]$} \\
\hline 21 & 28 & 35 & 35 & 28 & 28 \\
\hline \multicolumn{6}{|c|}{ In6 $\left[\mathrm{kW} / \mathrm{m}^{2}\right]$} \\
\hline 35 & 42 & 35 & 35 & 42 & 35 \\
\hline \multicolumn{6}{|c|}{$\operatorname{In} 7\left[\mathrm{~kW} / \mathrm{m}^{2}\right]$} \\
\hline 56 & 63 & 56 & 56 & 63 & 56 \\
\hline
\end{tabular}

Calculations were conducted for six cases, selected on the basis of the mass flow values at the inlet (DP0DP5, table 2). The values of the average velocity and the fluid temperature were defined as the inlet boundary condition on the "Inlet" surface. These values were selected based on the results of experimental studies. The same way was used for defining the pressure in the system, for each point. On the basis of the radiation schemes, obtained using the model used in accordance with the RTMC method, also the boundary conditions were defined on the absorber's surface, divided into 7 rings. The heat flux derived from the radiation reflected by the concentrator incident on the absorber's surface was lowered by $30 \%$ of its value in order to obtain the complete heat flux passing through the surface.

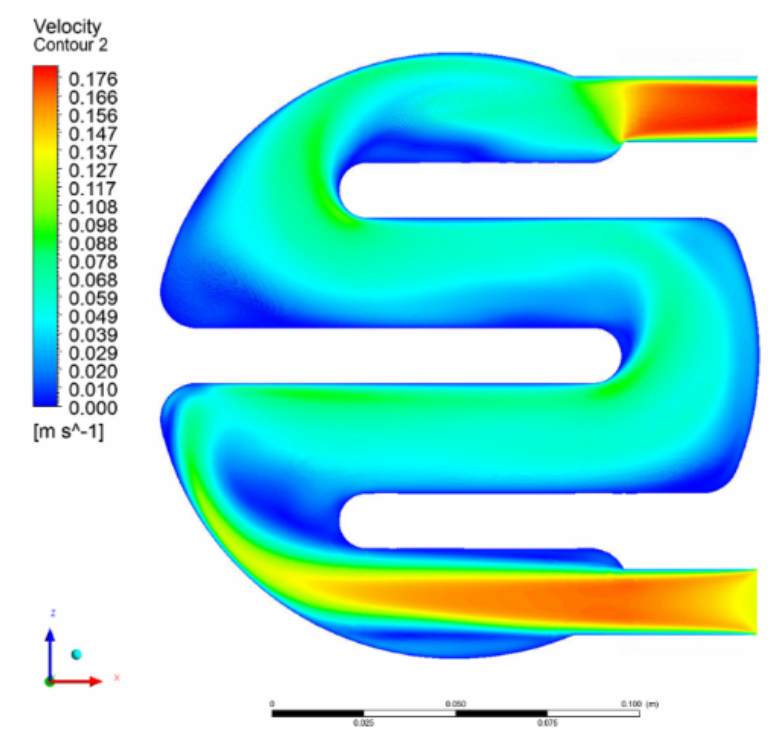

Fig. 11 Average velocity value at symmetry cross-section plane of fluid domain for DP0 


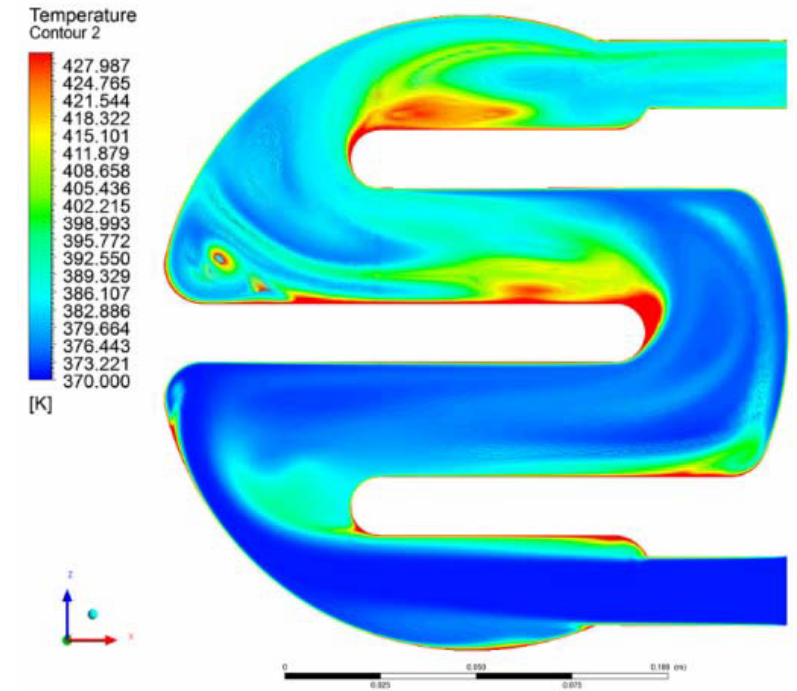

Fig. 12 Temperature distribution at symmetry cross-section plane of fluid domain for DP0

Fig. 11 shows the average value of the velocity on the symmetry surface of the fluid domain. Some zones, in which the oil flow is inefficient, and the average speed is near 0 can be observed on the bends of the channel. These zones are the largest in the area of the first and the third return of the fluid. Figure 12 in turn shows the temperature distribution on the same symmetry plane. It can be observed how these two parameters relate to each other. Due to the insufficient heat transfer in the areas, where the flow velocity is close to zero, the temperature value is clearly higher. In certain areas (in the middle part of the receiver) these values are even almost $100 \mathrm{~K}$ higher than the average temperature value of the fluid. This phenomenon not only affects the reduction of the efficiency of the heat exchange, but it can cause the local sources of overheating of the fluid. The results of numerical modelling therefore suggest that some modifications of geometry, which aim to align the flow, could have a positive impact on the increase of the efficiency of the receiver.

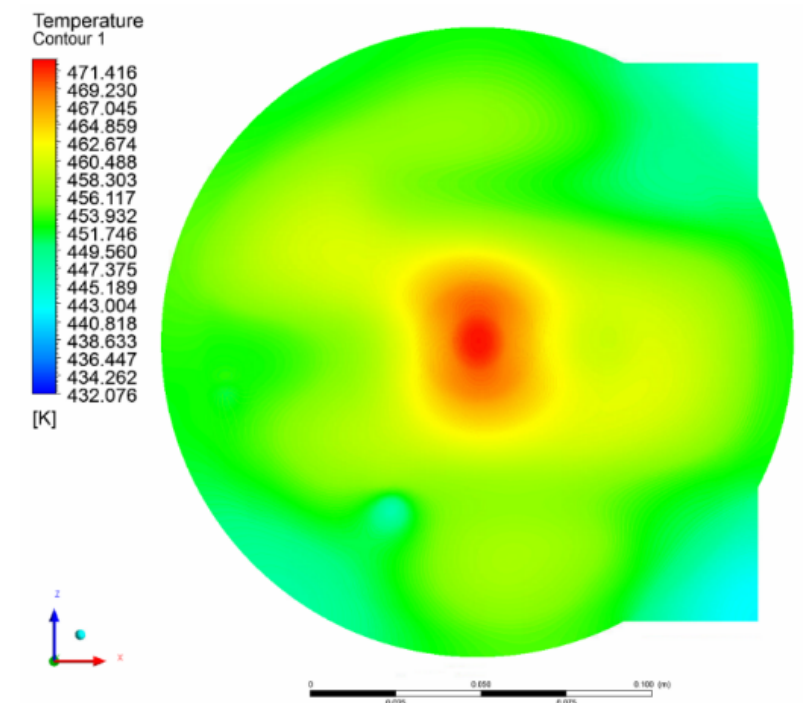

Fig. 13 Temperature distribution at the surface of absorber for DP0
Figure 13 presents the temperature distribution on the absorber's surface. These values are very difficult to measure in the experimental manner, due to the large impact on the operation of measuring instruments falling on the surface of solar radiation. The average temperature on the surface of the absorber was $460 \mathrm{~K}$. The highest value obtained in the vicinity of the focal point of the concentrator and exceeded $470 \mathrm{~K}$.

\section{Discussion}

As part of the research the experimental measurements were performed using the installations of the solar collectors connected with the solar radiation collector, and calculations of the system using the numerical methods, RTMC and CFD.

As a result of experimental studies, the potentially most beneficial location of the receiver was determined in the geometrical system of the concentrator. For two selected distances the energy parameters of the system were measured, based on which the receiver's capacity was calculated.

The results obtained in the way of the experiment were then used as the input data for conducting computer simulations with the Ray Tracing Monte Carlo method. This way the values of the intensity of the radiation absorbed by the surface of the high-temperature heat receiver was determined. These values were then used as boundary conditions for creating the numerical model with the Computational Fluid Dynamics method.

The completed research works allowed the performance of the validation of the results of the computer simulations and the ones obtained through the experimental research. The results were presented in a graph in fig. 14.

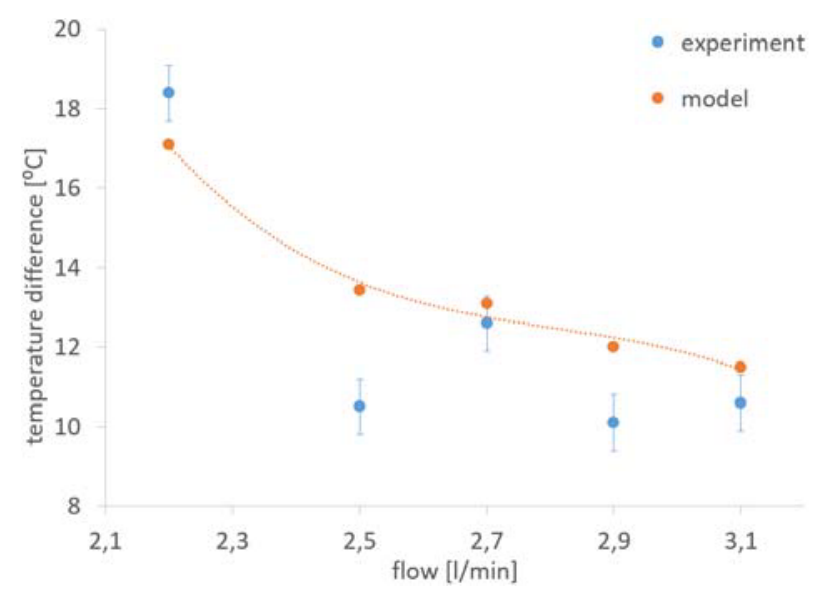

Fig. 14 Temperature at the surface of absorber placed at the distance of $76 \mathrm{~cm}$ from the mirror surface

The graph in fig. 14 presents the values of temperature difference between the outlet and inlet obtained for different values of the working medium flow through the receiver, obtained in way of the experiment and numerical modelling. The highest values of reheating the medium obtained in the case of the volumetric stream equal approx. $2.2 \mathrm{l} / \mathrm{min}$. Because of the varied atmospheric conditions, the power at this 
point was lower than the power obtained for the flow contained in the range of $2.5-3 \mathrm{l} / \mathrm{min}$ (fig. 6). However, the studies showed that when using the system of the concentrator it is possible to increase the temperature of the factor by nearly $20^{\circ} \mathrm{C}$, what is sufficient to effectively power the absorption chiller.

The studies conducted in diverse approaches (experimental measurements and computer simulations) made it possible to optimise the hybrid system of solar collectors with the solar radiation concentrator. On the path of the experimental studies and simulations of the Ray Tracing type it was indicated that the best location of the receiver in the geometrical system of the concentrator is the distance of $76 \mathrm{~cm}$ from the mirror's surface. The comparison of the experimental results with the numerical model implemented with the CFD method allowed to point out that the best value of the working medium flow for the studied system is approx. $21 / \mathrm{min}$.

Further researches will be related to cool production in sorption chiller powered by high temperature heat generated in concentrated solar radiation system.

\section{Acknowledgement}

The work has been completed as part of the statutory activities of the Faculty of Energy and Fuels at the AGH University "Studies concerning the conditions of sustainable energy development".

\section{References}

[1] A. M. Daabo, S. Mahmouda, R. K. Al-Dadah, Appl. Energ. 179, 1081-1096, (2016)

[2] R. Perez-Enciso, A. Gallo, D. Riveros-Rosas, E. Fuentealba-Vidal, C. Perez-Rábago, Renew. Energ. 93, 115-124, (2016)

[3] M. Rahou, A. Mojiri, G. Rosengarten, J. Andrews, Sol. Energy, 124, 15-25, (2016)

[4] M.I. Roldán a, E. Zarza, J.L. Casas, Renew. Energ. 76, 608-618, (2015)

[5] N. Tu, J. Wei, J. Fang, Sol. Energy 112, 128-143

(2015)

[6] M.I. Roldán, O. Smirnova, T. Fend, J.L. Casas, E. Zarza, Renew. Energ., 62, 116-128, (2014)

[7] E. Przenzak, M. Szubel, M. Filipowicz, Energ. Convers. Manage., 125, 97-106, (2016)

[8] E. Przenzak, M. Filipowicz, EPJ - Web of Conferences, 114, $02100 \quad$ (2016), DOI: http://dx.doi.org/10.1051/epjconf/201611402100

[9] E. Bożek, M. Filipowicz, J Civil Eng. Env. Arch., 32, vol.62 no2, 19-28, (2015)

[10] TracePro software, Lambda Research Corporation, http://www.lambdares.com/, last access 06.10.2016

[11] Allen C. W., Astrophysical Quantities, The Athlone Press, London, 1963

[12] ANSYS software, Inc, http://www.ansys.com/, last access 06.10 .2016 